\title{
Enhanced Spin-Polarized Transportation Through DNA by Quantum Topological Effect
}

\author{
Subhamoy Singha Roy \\ Department of Physics, JIS College of Engineering (Autonomous), West Bengal University of Technology, Kalyani, Nadia, India \\ Email address: \\ ssroy.science@gmail.com, subhamoy.singharoy@jiscollege.ac.in

\section{To cite this article:} \\ Subhamoy Singha Roy. Enhanced Spin-Polarized Transportation Through DNA by Quantum Topological Effect. Biomedical Statistics and \\ Informatics. Vol. 5, No. 3, 2020, pp. 70-75. doi: 10.11648/j.bsi.20200503.13
}

Received: June 12, 2020; Accepted: July 3, 2020; Published: September 7, 2020

\begin{abstract}
We report on the nature of the DNA molecule is a promising candidate for molecular Polarization in the Quantum framework of Berry phase factor. Our analysis is based on the polarization of states of various quantum system in lowest Landau level and also the dynamical machine which predicts nonadiabaticity in the neighborhood of the critical point. It is now detected that the low energy excitations states for a completely polarized state of a quantum structure is a soliton where as for unpolarized states soliton excitation are not imaginable. Aimed at incompletely polarized states also skyrmion excitations do not appear to happen as the skyrmionics solitons. We have studied the order of quantum states from the interpretation fact of chiral anomaly segments of DNA molecule and Barry Phase. However, later the series of mutual critical behaviour looks to be very narrow. The physics succeeding the quantum skyrmions is considered here from the understanding point of quantum topological partition. We also observed that skyrmions (soliton) are the pertinent DNA molecule states with filling factor $v=1$ it is fermion and also accomplished when we have DNA molecule hole conjugate states given by $v=1 /(2 m+1)$. A hole configuration is pronounced by the compound conjugate of the DNA molecule state, the spin alignment of the molecule and hole state will be reverse to individually added.
\end{abstract}

Keywords: DNA Molecules, Berry Phase, Polarization

\section{Introduction}

In this note we shall study the two polynucleotide chains are coiled about the same axis with a specific helical sense in a DNA molecule, we may visualize it such that a spin with a specific orientation is inserted on the axis indeed as the rotation of the two strands about the axis in the positive direction through an angle $\pi$ is identical with that of rotation about the axis in the negative direction through an angle $\pi$, we can uplift the rotation group $S O(3)$ to $S U(2)$. A spin is represented as an $S U(2)$ gauge bundle [1]. In view of this we can consider that twisting of the two strands can be taken to be represented by a spin inserted on the axis such that two adjacent coils have opposite orientations of the spin. This essentially implies that two strands twisting about the axis in the opposite direction can be designated by two spins having orientations $+1 / 2$ and $-1 / 2$. When these spins are inserted on the axis such that two spins having opposite orientations are located in the two adjacent coils with lattice spacing of one period of helix this represents an antiferromagnetic spin chain. The nearest neighbor spin -spin interaction can be viewed as to represent the twisting energy.. We have shown in some earlier papers $[1,2]$ that a spin can be represented by an $S U(2)$ gauge bundle and we can study a spin system in terms of these gauge fields. Here we shall show that when the conformational properties of DNA are mapped onto a Heisenberg spin chain we can represent the topological properties such as the linking number in terms of these gauge fields. Now in quantum reflex entanglement of two spin $1 / 2$ particles the Berry phase plays an important role during spin spin interactions (spin -echo) method [3, 4]. Kitaev described [5] the topological and quantum computer as a mechanism in which quantum information carried by electron-hole residing in two dimensional (2D) electron gas have long range Aharonov-Bohm (AB) connections between one another. These $\mathrm{AB}$ interactions are responsible for nontrivial phase values during interwinding of electron-hole trajectories in course of time evolution of Quantum topological Effects. Here we suggest to study the a mixture of polarization states of quantum skyrmions on the basis of this hierarchical model 
also we will plan at perceptive the DNA molecule spin polarization of various quantum system in the lowest Landau level $v=1$ and then parent states $v=1 /(2 m+1)$ from the view point of geometric quantum calculation.

\section{DNA Molecules and Polarization Quantum Model}

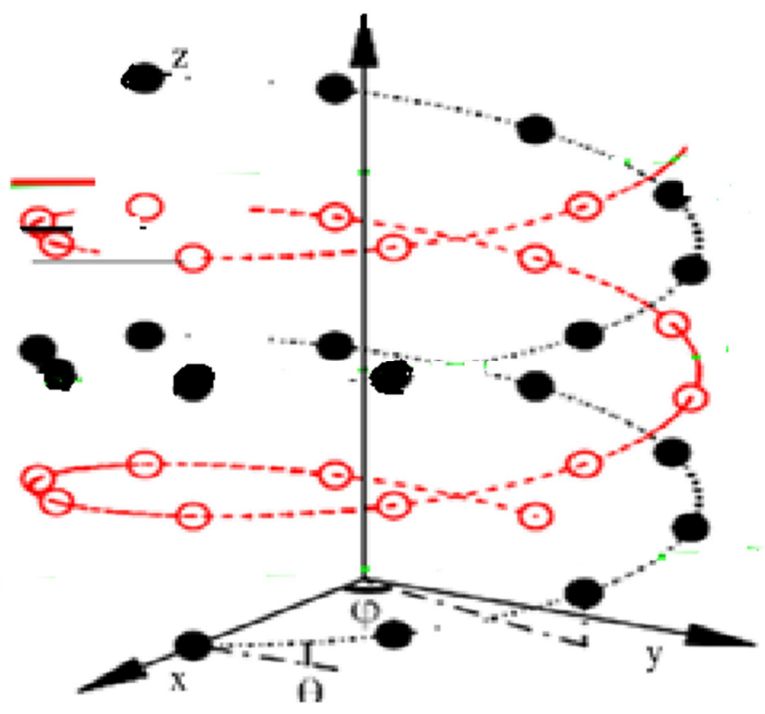

Figure 1. Diagram interpretation of DNA molecule- right-handed with double helix angle $\theta$ and cylindrical coordinate $\varphi$ under radial magnetic field. another DNA molecule - left-handed can be consequent by substituting $\theta$ with $\pi-\theta$ and $\varphi$ with $-\varphi$.

In the depiction of DNA molecule as an antiferromagnetic spin chain we observe that the spin degree of freedom appears to be associated with three Euler angles $\theta, \phi$ and $\psi$ corresponding to an extended body. In fact it has been shown in earlier papers $[6,7]$ that the quantization of a fermion in the framework of Nelson's stochastic quantization procedure [8] can be achieved when we introduce an internal variable which appears to be a direction vector depicting the spin degrees of freedom. The direction vector effectively corresponds to a vortex line which is topologically equivalent to a magnetic flux line. When we introduce the direction vector attached to a space-time point the spherical harmonics associated with the system incorporates apart from the polar angles $\theta$ and $\phi$, another angle $\psi$ which is related to the rotational orientation of the direction vector. These three angles correspond to three Euler angles associated with an extended body. Thus the spin degrees of freedom introduces three Euler angles in the system. Indeed as a spin degree of freedom appears as $S U(2)$ gauge bundle and the group $S U(2)$ is topologically equivalent to the 3 -sphere $S^{3}$, we note that $S^{3}$ incorporates an extended body having three Euler angle $\theta, \phi, \psi$. It is observed that the spherical harmonics incorporating these three Euler angles corresponds to monopole harmonics as the eigenvalue of the operator $i \hat{\partial} / \partial \psi$ corresponds to a monopole charge. Indeed we can write the corresponding harmonics as $Y_{l}^{m, n}, m(\mu)$ being the eigenvalue of the operator $\left.i \hat{\partial} / \partial \phi^{(i} \hat{\partial} / \partial \psi\right), l$ being the angular momentum [9]. Here in geometry, electrons are placed on the surface of a sphere under the influence of an uniform radial magnetic field Figure 1. The magnetic field is produced by a magnetic monopole of strength $\mu$ placed at the centre of the sphere. As we know, the angular momentum in the field of a magnetic monopole is given by:

$$
\begin{gathered}
\vec{J}=\left[(\hat{i} x+\hat{j} y+\hat{k} z) \times\left(\hat{i} P_{x}+\hat{j} P_{y}+\hat{k} P_{z}\right)\right]-\mu \hat{a} \\
\vec{J}=(\vec{r} \times \vec{P})-\mu \hat{a}
\end{gathered}
$$

where $\mu$ can take the values $0, \pm 1 / 2, \pm 1, \pm 3 / 2$ Evidently, eigen values of $\mathrm{J}$ can take the values $|\mu|,|\mu|+1,|\mu|+2$,

The spherical harmonics incorporating the term $\mu$ have been extensively studied by Fierz [10] and Hurst [11]. Following them we write

$$
Y_{l}^{m \cdot \mu}=(1+x)^{-(m-\mu) / 2}(1-x)^{-(m+\mu) / 2} \frac{d^{l-m}}{d_{x}^{l-m}}\left[(1+x)^{l-\mu}(1+x)^{l+\mu}\right] e^{i m \phi_{e}-i \mu \chi}
$$

Where $x=\cos \theta$ and the quantities $\mathrm{m}$ and $\mu$ just represent the eigenvalue of the operators $i(\partial / \partial \phi)$ and $i(\partial / \partial \chi)$. As mentioned in introduction division a spin may be depicted in terms of fermionic oscillators. We can depict a twocomponent spinor as $\left[\begin{array}{l}u \\ v\end{array}\right]$ with [12]

$$
\begin{aligned}
& u=Y_{1 / 2}^{1 / 2,1 / 2}=\operatorname{Sin} \theta / 2 e^{[i(\phi-\chi) / 2]} \\
& v=Y_{1 / 2}^{-1 / 2,1 / 2}=\operatorname{Cos} \theta / 2 e^{[-i(\phi-\chi) / 2]}
\end{aligned}
$$

In terms of the spin system we can consider the ground state wave function depicting the DNA supercoil

$$
\left|\psi_{v=0}\right\rangle=\prod_{i\langle j}\left(u_{i} v_{j}-u_{j} v_{i}\right)
$$

where $i$ and $j$ correspond to the spin sites. Where $v=1 / m$, $m$ being an odd integer. When the spins begin to be tilted just below the critical point, the resulting skyrmion state is described by

$$
|\psi\rangle=C \prod_{k}\left(\begin{array}{c}
v_{k} \\
-\alpha u_{k}
\end{array}\right)\left|\psi_{0}\right\rangle
$$

where the spin texture is included within the components $v_{k}$ and $u_{k}$ with $0 \leq \alpha \leq 1$ [13].

It is noted that when $\mu$ is an integer, we can have a relation of the form 


$$
\vec{J}=(\vec{r} \times \vec{P})-\mu \hat{a}=A+B
$$

This indicates that the Berry phase which is connected with $\mu$ may be unitarily removed to the dynamicalphase. Evidently, the average magnetic field may be considered to be vanishing in these states. The attachment of $2 m$ vortices to an electron effectively leads to the removal of Berry phase to the dynamical phase.

$$
\begin{aligned}
& \text { When } \vec{r} \times \vec{P}=0 \\
& \Rightarrow \vec{J}=-\mu a
\end{aligned}
$$

and $\mu_{\text {eff }}=3 / 2$ this implies the ground state for the Landau lavel. So, DNA molecule quantum states with $2 \mu_{e f f}=2 m+1$ ( $\mathrm{m}$ an integer)

$$
\text { Now we can write } 2 \mu_{\text {eff }}=C+1
$$

with $C=2 m n$ and filling factor as

$$
v=\left(n / 2 \mu_{e f f}\right)=1 /(C \pm 1)
$$

In this scheme the DNA molecule -hole conjugate states

$$
v=1-n /(C \pm 1)=n^{\prime} /\left(2 m n^{\prime} \pm 1\right)
$$

Where $n$ is an odd and $\quad n^{\prime}$ is an even integer.

During the representation of DNA molecule as an antiferromagnetic spin chain, Bouchiat and Me'zard [14] have critical out that the writhe partition function Fourier transform corresponds to the quantum mechanical problem of a charged particle in the field of a monopole with nonquantized charge. These authors have prescribed a local writhe formula in terms of the Euler angles $\phi(s)$ and $\psi(s)$ defining a line integral

$$
\chi=\phi(L)+\psi(L)=\int_{0}^{L} d s(\dot{\psi}+\dot{\phi})
$$

Where $L$ is the total number of spins in the chain and $L / 2$ is the number of singlets.

The total twist can be written as

$$
T w=\int_{0}^{L} d s(\dot{\psi}+\dot{\phi} \cos \theta)
$$

A "local writhe" $\chi W$ is defined as

$$
\chi W=\chi-T w=\int_{0}^{L} d s \dot{\phi}(1-\cos \theta)
$$

They have shown that $\chi W$ can be written as the line integral $\int d \phi A_{\varphi}=\int \vec{A}_{m}(\vec{r}) d \vec{r}$ where $A_{\phi}=(1-\cos \theta)$ is identified as the $\phi$-component of the potential vector $\vec{A}_{m}(\vec{r})$ of a magnetic monopole of charge unity. In fact for a closed circuit we have the relation

$$
\int_{0}^{2 \pi} A_{\phi} d \phi=2 \pi(1-\cos \theta)
$$

It is observed that the holonomy given by eqn.(17) effectively corresponds to the Berry phase [15] acquired by a quantum state while traversing a closed path. In fact the Berry phase acquired by a spin state in a spin $1 / 2$ chain when the Hamiltonian is parallel transported along a closed circuit is given by $[16,17]$

$$
\phi_{B}=\pi(1-\cos \theta)
$$

It is noted that the holonomy given by eqn.(17) is twice this phase factor. The effective monopole charge associated with a spin state in an entangled spin system is given by the relation

$$
\mu_{\text {eff }}=(1 / 2)(1-\cos \theta)
$$

which follows from the relation $\phi_{B}=2 \pi \mu[18,19]$. Evidently the monopole charge takes nonquantized value apart from the situation when the polar angle $\theta$ of the spin axis with the quantization axis is given by $\theta=0, \pi / 2$ and $\pi$. Now to study the DNA molecule polarization states of various quantum systems, we observed that in the lowest Landau level where $(\mathrm{n}=1)$, we have the filling factor $v$ given by

$$
v=\left(n / 2 \mu_{e f f}\right)=\left(1 / 2 \mu_{e f f}\right)
$$

From eqn. (19)

$$
\begin{gathered}
\mu_{e f f}=(1 / 2)(1-\cos \theta) \\
\Rightarrow 2 \mu_{e f f}=(1-\cos \theta) \\
\text { So } v=\left(1 / 2 \mu_{e f f}\right)=[1 /(1-\cos \theta)]=1 /(2 m+1)
\end{gathered}
$$

Since we have keen out that the connection of $2 \mathrm{~m}$ vortices to an electron leads to the elimination of Berry phase to the dynamical phase and this successfully corresponds to the accessory of one vortex to an electron, this electron will exist a polarized individual.

\section{DNA Molecule Spin Polarazed of Skyrmions}

To study through in the quantum framework of $3+1$ topological dimensional manifold in Pure Sigma model. In this structure, the quartic steadiness term introduced by 
Skyrme, identified as the Skyrme term, determines the size of the skyrmions. In this systems deviations from the close filling factor $v$ is able by the squalor of the systems spin polarization $[20,21]$. Here in geometry, electrons are placed on the surface of a sphere under the influence of an uniform radial magnetic field. The magnetic field is produced by a magnetic monopole of strength $\mu$ placed at the centre of the sphere. As we know, the angular momentum in the field of a magnetic monopole is given by the eqn (2), Now from eqn.(6) given by the smooth and monotonical function $g(\theta)$ is defined with $g(0)=0$ and $g(\pi)=\pi$ then the skyrmion state can be written as

$$
\vec{\phi}(\Omega)=\cos (g(\theta)-\theta) \vec{e}_{r}+\sin (g(\theta)-\theta) \vec{e}_{\theta}
$$

where $\vec{e}_{r}$ and $\vec{e}_{\theta}$ are the basis vectors. The size of a skyrmion is determined by the function $g(\theta)$ and $g(\theta)=\theta$ describes the hedgehog skyrmion with spin in the radial direction $\vec{r}$.

The skyrmion state $\vec{\phi}(\Omega)$ is constrained by the relation $|\vec{\phi}(\Omega)|=1$. The quantum state for the skyrmion $\vec{\phi}(\Omega)$ can be written as

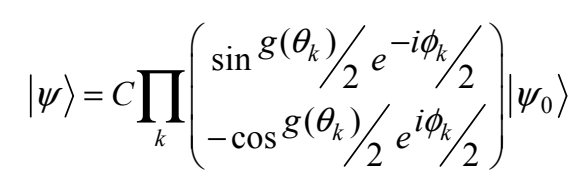

where $C$ is the normalization constant and $g(\theta)$ controls the size of the skyrmion. From eqns. (5) and (6) it is seen that $\alpha$ is determined from $g(\theta)$ and as such it controls the size of the skyrmion.

Indeed we can define

$$
\theta=2 \arctan \alpha
$$

which equals $\pi / 2$ for the hedgehog skyrmion with $\alpha=1$. Bychkov et. al. have shown [22] that the quantum aspects skyrmions consist of a core whose size is defined by the interplay between the Zeeman and Coulomb energies and an additional length scale $B_{s k}$ which determines the tail of the spin distribution. This characteristic length is given by

$$
B_{s k}^{-2}=2 \sqrt{\frac{2}{\pi} \mid \tilde{g}\left(c_{B} \mid / l^{3}\right)}
$$

where $g^{\sim}$ is the effective $g$ factor, $c_{B}=\varepsilon \hbar^{2} / m e^{2}$ is the Bohr radius, $\varepsilon$ being the dielectric constant and $l$ is the magnetic length. It is noted that as $g^{\sim} \rightarrow 0, B_{s k} \rightarrow \infty$ we can take

$$
\cot (\theta / 2)=x / x_{0}
$$

where $x$ and $x_{0}$ are two dimensionless parameters given by $x=r / B_{s k}$ and $Y=x_{0} / B_{s k}$, Y being the size of the skyrmion core region. Taking the spin variable $\vec{z}=U \vec{z}_{0}$ with $\vec{z}_{o}=\left(\begin{array}{l}1 \\ 0\end{array}\right)$ and $U \in S U(2)$ we may write the nonlinear $\sigma$-model Lagrangian in terms of the $S U(2)$ matrices $U$ as [23]

$$
L=-\left(M^{2} / 16\right)\left(\partial_{u} U^{?} \partial_{\mu} U\right)-\left(1 / 32 \eta^{2}\right)\left(\partial_{\mu} U U^{?}, \partial_{\nu} U U^{\dagger}\right)^{2}
$$

where $M$ is a constant having dimension of mass and $\eta$ is a dimensionless parameter, $\mu, v$ being space -time indices. The $\alpha$ dependence may be incorporated through $M$ and $\eta$ where these parameters are taken as functions of $\alpha$.

We can consider the radius of the DNA loop $Y$ (plectoneme radius) as a function, $Y(\theta, \phi)$ corresponding to the core radius of the skyrmion. We can define the core size of the skyrmion such that $Y=Y_{0}(1-\alpha)$ where $Y_{0}$ is the size of the skyrmion with minimal energy. In fact in an axisymmetric system where the anisotropy is introduced along a particular direction through the introduction of a magnetic monopole at the centre, the components of the linear momentum satisfy a commutation relation of the form.

$$
\left[z_{i}, z_{j}\right]=i \mu \varepsilon_{i j k} \frac{x^{k}}{r^{3}}
$$

When the position space is a 3 -sphere $S^{3}$ with a monopole at the centre, we can have a commutation relation of the form

$$
\left[z_{i}, z_{j}\right]=(i / Y) \varepsilon_{i j k} z_{k}
$$

where $\mathrm{Y}$ is proportional to the radius of the $S^{3}$. For a distorted sphere we can consider $R$ as a functional form $Y(\theta, \phi)$ corresponding to the core radius of the skyrmion. We can define the core size of the skyrmion such that $Y=Y_{0}(1-\alpha)$ where $Y_{0}$ is the size of the skyrmion having minimal energy.

In view of this, in $3+1$ dimensions we can generalize the Lagrangian in terms of the Skyrmion as

$$
\begin{aligned}
& L=2 \pi J_{S}^{\mu}\left(A_{\mu}+A_{\mu}^{0}\right)-\frac{1}{2} \theta \varepsilon^{\mu v \sigma} A_{\mu} F_{v \sigma} \\
& =\frac{\lambda}{4} F_{\mu \nu} F^{\mu \nu}-\frac{1}{8 \lambda}(\nabla n)^{2}
\end{aligned}
$$

Where the field strength term $F_{\mu \nu}=\partial_{\mu} A_{v}-\partial_{\nu} A_{\mu}$ to be the duel of the electron number current, the skyrmion number current

$$
J_{\mu}^{S}=\frac{1}{2 \pi i} \varepsilon^{\mu v \sigma} \partial_{\mu} \tilde{z}_{\sigma} \partial_{\nu} z_{\sigma}
$$

It is well-known that the skyrmion number current acts as a source for a topologically massive gauge field [24] and also sees the background field $A_{\mu}$, and $\nabla=\left(\partial_{1} n / \partial_{2} n\right)$. Here $\varphi=(\varphi 1, \varphi 2)$ is a two component complex scalar field. $\theta$ is the statistics parameter which takes the values $\theta=(2 n+1) \pi$ so 
that the boson field $\varphi$ represents a fermion, $A_{\mu}$ the external electromagnetic field and $m^{*}$ is the effective mass of the electron. To determine the size and energy of the skyrmions Zeeman and Coulomb interactions should be included. However, to study the topological features we can ignore them for the moment. We consider a solution with uniform density $\rho=(|\varphi 1| 2+|\varphi 2| 2)=\rho_{0}$ at filling fraction $v=1$ and $v=1 /(2 m+1)$. Now the adjusting the unit of length and time such that $c=\sqrt{\lambda \rho_{0} / m^{*}}$, the velocity of density waves in the absence of magnetic field becomes unity.

Now we can generalize the Lagrangian eqn. (28) absorbing Skyrme term in the form [25]

$$
\begin{gathered}
L=2 \pi J_{S}^{\mu}\left(A_{\mu}+A_{\mu}^{0}\right)-\frac{M^{2}}{16} \operatorname{Tr}\left(\partial_{\mu} U^{?} \partial_{\nu} U\right)-\frac{1}{32 \eta^{2}} \operatorname{Tr}\left(\partial_{\mu} U U^{\dagger}, \partial_{\nu} U U^{\dagger}\right)^{2} \\
-\frac{\theta}{16 \pi^{2}}{ }^{*} F_{\mu \nu} F_{\mu \nu}-\frac{\lambda}{4} F_{\mu \nu} F^{\mu \nu} \\
\text { Where }{ }^{*} F_{\mu \nu}=\frac{1}{2} \varepsilon_{\mu v \alpha \sigma} F_{\lambda \sigma},
\end{gathered}
$$

where $A_{\mu}\left[A_{\mu}^{[0]}\right]$ the static nonlinear $\sigma-$ model Lagrangian corresponding to eqn (33) gives rise to the energy, the kinematic term, the second term in eqn (17) which takes into account spin waves.

Now we may write nonlinear $\sigma$ - model, the $S U(2)$ matrices $U$ as $U=n_{0} I+n \cdot \vec{\tau}$, in this model $n_{i}(\mathrm{i}=1,2,3)$ corresponding to this spin direction which live on the $2 \mathrm{D}$ surface of the sphere. Now we can construct the fallowing algebra.

$$
\begin{gathered}
{\left[m_{i}, m_{j}\right]=i \varepsilon_{i j k} m_{k}} \\
{\left[m_{i}, n_{j}\right]=i \varepsilon_{i j k} n_{k}} \\
{\left[n_{i}, n_{j}\right]=i \varepsilon_{i j k} m_{k}} \\
L_{i}=(1 / 2)\left(m_{i}-n_{i}\right) \\
Y_{i}=(1 / 2)\left(m_{i}-n_{i}\right) \\
{\left[L_{i}, L_{j}\right]=i \varepsilon_{i j k} L_{k}} \\
{\left[Y_{i}, Y_{j}\right]=i \varepsilon_{i j k} Y_{k}} \\
{\left[L_{i}, Y_{j}\right]=0}
\end{gathered}
$$

Thus the algebra has split into two independent subalgebras each isomorphic to a $S U(2)$ algebra corresponds the chiral group $S U(2)_{L} \otimes S U(2)_{R}$. The left and right chiral group can now be taken to be associated with two DNA molecule mutually opposite orientations of the magnetization vector which resides on the $2 \mathrm{D}$ surface of the sphere. The chirality associated with twisting strain into the DNA molecule (pontryagin index) given by [26].

$$
q=2 \mu=-\frac{\theta}{16 \pi^{2}} \int \operatorname{Tr}^{*} F_{\mu \nu} F_{\mu \nu} d^{4} x
$$

Now the $2+1$ dimensions,

So the DNA molecule topological current

$$
J_{\mu}=\frac{1}{8 \pi} \varepsilon_{\mu v \lambda} n .\left(\partial_{v} n \times \partial_{\lambda} n\right)
$$

The gauge field current

$$
J_{\mu}=\varepsilon_{\mu \nu \lambda} \nabla_{v} A_{\lambda}
$$

Which help us to write the Chern-Simons term as

$$
W=\frac{\theta}{4 \pi} \int d^{3} x \varepsilon_{\mu \nu \lambda} A_{\mu} \nabla_{v} A_{\lambda}
$$

As the skyrmion (soliton) depicting a loop is described by the nonlinear $\sigma$-model in terms of $S U(2)$ gause fields, the topological charge of a DNA molecule is given by the winding number of the mapping of the 3-Space manifold into the group manifold $S U(2)=S 3$, which correspond to homotopy $\pi 3(S U(2))=\pi 3(S 3)=D$

$$
D=\frac{1}{24 \pi^{2}} \int_{S^{3}} d S_{\mu} \varepsilon^{\mu \nu \lambda \sigma}\left[\left(U^{-1} \partial_{\nu} U\right)\left(U^{-1} \partial_{\lambda} U\right)\left(U^{-1} \partial_{\sigma} U\right)\right]
$$

Indeed, the flux through the sphere is $2 \mu$ and the phase is given by $e^{i \varphi} B$ where $\varphi_{B}=2 \pi v$ (number of flux quanta enclosed by the DNA molecule). When a $D=1$ skyrmion is moved around a closed DNA molecule it acquires a Berry phase $2 \pi \nu n$ where $\mathrm{n}$ - is the number of skyrmions enclosed by the DNA molecule. To find when DNA molecule supercoil the linking number is given by an integer determined by this homotopy group so that $L k=n D$, the spin of the skyrmion having charge $v e D$ is given by $v D / 2$.

\section{Discussion}

So we have observed here that the spin precession as the charges $(q)$ eqn. (43) move from one base to another along only single channel DNA molecule. Now in this case spin orientation is Up or Down. As a result for the chiral group $S U$ $(2)_{L} \times S U(2)_{R}$ only one $S U(2)_{L}\left(S U(2)_{R}\right)$ group will be operative. From the homotopic relation $\pi_{3}(S U(2))=\pi_{3}(S$ $(3))=D$, Where D represent the set of integers, we will have solitons (skyrmions) with charge veD. So the states are fully polarised states $[18,27,28]$. Now in case unpolarized states number of down spin electrons is equal to the number of up spin electrons, in this case the homotopic relation $\pi_{3}(S U(2) \times$ $S U(2))=\pi_{3}(S O$ (4) $)=0$, we note that there will be no skyrmions. We find that skyrmions (soliton) are the relevant DNA molecule only at the filling fraction $v=1$ it is fermion 
(electron) and $v=1 /(2 m+1), \mathrm{m}$ being an integer represent fully polarized states.

\section{Funding}

None.

\section{Conflicts of Interest}

The author declares there is no conflict of interest.

\section{Acknowledgements}

The authors are grateful to express my deep gratitude to my beloved Sir Prof. Pratul Bandyopadhyay for helpful discussion.

\section{References}

[1] P. Bandyopadhyay, Proc. Roy. Soc (Londan) A. 466, 2917 (2010).

[2] G. Goswami and P. Bandyopadhyay, J. Math. Phys. 34, 749 (1995).

[3] V. Vedral, Cent. Eur. J. Phys. 2, 289 (2003); q uantphys/0505029. V. Vedral, quantum-ph/0212133.

[4] D. Banerjee; Phys. Rev.-B58, 4666 (1998).

[5] A. Y. Kitaev; Ann. Phys. 303, 2 (2003).

[6] P. Bandyopadhyay and K. Hajra: J. Math. Phys. 28, 711 (1987).

[7] K. Hajra and P. Bandyopadhyay: Phys. Lett. A 155, 7 (1991).

[8] E. Nelson: Phys. Rev. 150, 1079 (1966); Dynamical theory of Brownian motion (Princeton University Press, Princeton, N. J. (1967).

[9] C. A. Hurst: Ann. Phys 50, 37 (1968).
[10] M. Fierz, Helv. Phys. Acta 17, 27 (1944).

[11] C. A. Hurst, Ann. Phys. 50, 51 (1968).

[12] B. Basu, Mod. Phys. Lett. B6, No. 25, 1601 (1992).

[13] B. Basu, S, Dhar and P. Bandyopadhyay, Int. J. Mod. Phys. B 18 (2004) 171.

[14] C. Bouchiat and M. Mezard: Phys. Rev. Lett. 80, 1556 (1997); Euro. Phys. J. E, 2, 377 (2000).

[15] M. V. Berry, Proc. Roy. Soc (Londan) A, 392, 45 (1984).

[16] B. Basu and P. Bandyopadhyay, Int. J. Geo. Meth. Mod. Phys. 9707 (2007).

[17] B. Basu and P. Bandyopadhyay, J. Phys. A. 41, 055301 (2008).

[18] P. Bandyopadhyay: Proc. Roy. Soc (London) A 467, 427 (2011).

[19] S. Singha Roy: Theoretical Physics, 2, Number 3, 141 (2017).

[20] E. H. Aifer, B. B. Goldberg and D. A. Broido, Phys. Rev. Lett. 76, 680 (1996).

[21] D. K. Maude, M. Potemski, J. C. Portal, M. Henini, L. Eaves, G. Hill and M. A. Pate, Phys. Rev. lett. 77, 4604 (1996).

[22] Yu. A. Bychkov, A. V. Kolesnikov, T. Maniv and I. D. Vagner, J. Phys. Cond. Matt. 10, 2029 (1998).

[23] T. H. R. Skyrme, Proc. Roy. Soc. A 260 (1962) 127, Nucl. Phys. 31 (1961) 556.

[24] M. Stone, Phys. Rev. B53, 16573 (1996).

[25] B. Basu, S. Dhar and P. Bandyopadhyay, preprint condmat/0208426.

[26] D. Banerjee and P. Bandyopadhyay, J. Math. Phys. 33, 990 (1992).

[27] S. S. Mandal and V. Ravishankar, Phys. Rev. B 57, 12333 (1998).

[28] S. Singha Roy and P. Bandyopadhyay,: Phys. Lett. A 382, 1973 (2018). 